\title{
hTERT rs2736098 Genetic Variants and Susceptibility of Hepatocellular Carcinoma with Chronic Hepatitis $C$ in the Egyptian Patients
}

\author{
HEBAT ALLAH M. SHARAF EL-DEEN, M.D.; ENAS H. MAHMOUD, M.D.; \\ AHMED A.E. HEKEL, M.D.; ALAA A. ABDEL RAUOF, M.D.; \\ SUZAN E. MAHROUS, M.D. and ASMAA E.A. NASR ELDIN, M.Sc.
}

The Department of Clinical and Chemical Pathology, Faculty of Medicine, Cairo University

\begin{abstract}
Background: Telomere length and telomerase activity are crucial for cancer initiation and the survival of tumors. Telomere length is maintained by telomerase in $90 \%$ of human cancers, while $10 \%$ of cancers use an alternative mechanism of telomere lengthening termed (ALT). Another mechanism for Cancer cells is through activating or up-regulating the normally silent human TERT gene (hTERT) that encodes telomerase.
\end{abstract}

Aim of Study: The aim of the present study was to assess the effect of a SNP in TERT gene rs2736098 on telomere length, furthermore, a study was conducted to investigate whether the changes of median values of Relative Telomere Length (RTL) is related to a SNP genotype.

Patients and Methods: The study was conducted on a total number of 100 patients, subdivided into two groups HCC and HCV groups. SNP was measuring using Taqman probe on step-one real time PCR.

Results: It was found that homozygous GG genotype in SNPrs 2736098 was high in both HCC and HCV comparing to heterozygous GA genotype and variant AA genotype. Moreover, the frequency of the SNP rs $2736098 \mathrm{G}$ allele compare to A allele was higher in HCV than $\mathrm{HCC} 82 \%$ versus $76 \%$ respectively.

Conclusion: There was no statistical significant difference observed on comparing a SNP rs2736098 in HCC and HCV groups and there was no statistically significant association was found $(p=0.69)$ when we investigated whether the changes of median values of RTL is related to a SNP genotype.

Key Words: Hepatocellular carcinoma - Relative telomeric length-Telomere maintenance gene.

\section{Introduction}

HEPATOCELLULAR cancer is the sixth most common cancer and the third leading cause of

Correspondence to: Dr. Hebat Allah M. Sharaf El-Deen, The Department of Clinical and Chemical Pathology, Faculty of Medicine, Cairo University death worldwide. It has a poor prognosis and the overall survival rates are 3-5\%. HCC has a mortality rate of $9.1 \%$ of global cancer [5] . In Egypt, HCC is the second most common cancer in men and the sixth most common cancer in women and it was reported that $\mathrm{HCC}$ accounts for about $4.7 \%$ of chronic liver disease with a remarkable increase in this proportion from $4.0 \%$ to $7.2 \%$ over the last decade [12]. This rising incidence may be due to a high prevalence of $\mathrm{HCV}$ and its complications. $\mathrm{HCV}$ infection is the main etiology of liver cancer in about $40-50 \%$ of cases [12]

Chronic infection with Hepatitis C virus is considered one of the major causes of liver cirrhosis and HCC. It affects 130-170 million people worldwide with high prevalence reported in Egypt [8] Egypt has one of the highest rates of HCV ( 15\%) worldwide [6].

Telomeres consist of repetitive sequences (TTAGGG) located at the ends of eukaryotic chromosomes which protect the integrity of genomic DNA [2]. Telomere length is maintained by telomerasein $90 \%$ of human cancers, while $10 \%$ of cancers use an alternative mechanism of telomere lengthening termed (ALT) [4]. Telomere shortening is a hallmark of cirrhosis and the main risk factor for the development of liver cancer through upregulation of hTERT gene that encodes telomerase [14] .

Telomeres protect chromosome ends from fusion and from being recognized as sites of DNA damage. Telomeres dysfunction arises from a critical shortening of telomeres in normal somatic cells during progressive cell divisions elicit DDRs that trigger cellular senescence [1]. 
Progressive shortening of telomere length may lead to senescence, apoptosis and oncogenic transformation of somatic cells which affects health and the life span of an individual [1].

Polymorphic variations such as SingleNucleotide Polymorphisms (SNPs) within several gene classes are considered to be important for regulation the length of telomeres and the higher order structure of the protein complex at the ends of linear chromosomes. Telomere maintenance genes are essential for maintaining genomic integrity and stability [3]. There are five telomere maintenance genes that affect tumor progression in HCC patients (TERT, TRF1, TRF2, POT1, TEP1) [7].

\section{Patients and Methods}

\section{Study design:}

This is cross-sectional study conducted at the Tropical Medicine Department and Internal Medicine Departments, Cairo University during the period from October 2015 to December 2016. Two groups of patients were enrolled: Group 1 (HCC group) included 50 patients with mean age of 56.74 \pm 7.9 , their diagnosis was based on their medical records and the presence of hepatic focal lesions diagnosed by abdominal ultrasound and confirmed by triphasic Computed Tomography (CT) according to American Association for the Study of Liver Diseases (AASLD) guidelines and there were also on top of HCV liver cirrhosis. Group 2 (HCV group) included 50 patients, their diagnosis was based on their medical records with mean age of $55.38 \pm 8.9$ years.

A written informed consent obtained directly from each subject before data collection and after explanation of the study objectives and the study protocol was approved by the Institutional review board of Cairo University.

\section{Biochemical investigations:}

Blood samples were collected and investigated for serum Alanine Transaminase (ALT), Aspartate Transaminase (AST), Alkaline Phosphatase (ALP), total bilirubin, albumin, creatinine, Alpha Feto Protein (AFP), Prothrombin Time (PT) and prothrombin International Normalized Ratio (INR), as well as complete blood count.

Selection of Candidate Genes: A SNP (rs2736098) was selected from telomerase reverse transcriptase for testing in this study. This SNP was chosen on the basis of previous reports of their association with the risk for HCC.
Laboratory methods: DNA was extracted from whole blood treated with EDTA using Gene JET DNA blood Mini kit-thermo Fisher Scientific according to the manufacturer's protocol. The concentration and purity of DNA were determined by measuring the absorbance at 260 and $280 \mathrm{~nm}$ using NanoDrop 1 000A Spectrophotometer (NanoDrop Technologies, Waltham, MA). Genotype identification was performed with Step One real-time PCR system (Applied Biosystems). Each PCR reaction mixture included: 5 the DNA, 10 TTMMan Universal PCR Master Mix (2x), No AmpErase UNG, 1 20 working stock of SNP Genotyping Assay and 4 Dase-free water to a final volume of 20 ung the following conditions: $95^{\circ} \mathrm{C}$ for $10 \mathrm{~min}, 95^{\circ} \mathrm{C}$ for 15 seconds min and $60^{\circ} \mathrm{C}$ for $1 \mathrm{~min}$. The SNP was identified by their position and fluorescent color in each well according to the position of the tagged oligonucleotides. Genotype data was generated on the basis of the relative fluorescent intensities for a SNP. The Sequence Detection System (SDS) Software used the fluorescence measurements made during the plate read to plot fluorescence $(\mathrm{Rn})$ values based on the signals from each well.

\section{Statistical methods:}

Statistical analysis was done using SPSS for Mac, release 24 (IBM Corporation, Armonk, NY, USA). Numerical data were expressed as mean and standard deviation or median (25 th- 75 th) as appropriate. Differences between groups are detected using Student's $t$ and Mann-Whitney tests as appropriate. Qualitative data were expressed as frequency and percentage. Chi-square test or Fisher's exact test was used to examine the relation between qualitative variables. Correlations between variables were done using Spearman's correlation test. All tests were two-tailed. A $p$-value $<0.05$ was considered significant.

\section{Results}

Characteristics of the study population: The baseline characteristics of the studied population in our study are shown in (Table 1).

Genotype and Allele frequency and effects on the development of HCC:

The patients with homozygous GG genotype in SNP rs2736098 was high in both HCC and HCV compared to heterozygous GA genotype and variant AA genotype, and $\mathrm{G}$ allele compared to A allele was higher in HCV than HCC (82\% versus $76 \%)$. However, a SNP showed no statistically significant difference between the two groups in the allele and genotype distribution. 
Table (1): Comparison of routine laboratory parameters of the studied groups.

\begin{tabular}{llll}
\hline Variables & HCC $(\mathrm{n}=50)$ & HCV $(\mathrm{n}=50)$ & $p$-value \\
\hline TLC X $10^{3} / \mu \mathrm{L}$ & $5.4(3.8-8.1)$ & $7.3(5.2-10.2)$ & 0.002 \\
Hb (gm/dl) & $11(9.6-12)$ & $9.65(8.5-10.6)$ & $<0.001$ \\
PLT X $10^{9} / \mathrm{L}$ & $129(88.5-160)$ & $123.5(78.7-194)$ & 0.926 \\
PT (sec.) & $15.7(14.1-17.5)$ & $17.5(14.9-22.2)$ & 0.025 \\
INR & $1.3(1.2-1.5125)$ & $1.4(1.2-1.6)$ & 0.297 \\
BUN (mg/dl) & $28.5(20-38.5)$ & $42(31-83)$ & $<0.001$ \\
Creat. (mg/dl) & $0.9(0.7-1.3)$ & $1.25(1-2)$ & 0.002 \\
AST (U/L) & $68.5(48.7-94.2)$ & $63.5(39.2-97)$ & 0.759 \\
ALT (U/L) & $36.5(21.7-51.2)$ & $37.5(19-51.7)$ & 0.888 \\
T. Bil (mg/dl) & $1.6(1-4.15)$ & $2.3(1.35-7.05)$ & 0.043 \\
D. Bil (mg/dl) & $0.5(0.3-1.7)$ & $1.05(0.4-2.95)$ & 0.063 \\
Albumin (g/dl) & $2.8(2.2-3.1)$ & $2.3(2-2.9)$ & 0.003 \\
ALP (U/L) & $103(72-183.2)$ & $81.5(59.5-102.2)$ & 0.015 \\
GGT (U/L) & $56(38.2-110)$ & $35(26.7-80)$ & 0.009 \\
AFP (ng/mL) & $12.95(5-99.3)$ & $15(4-51.2)$ & 0.241 \\
HCV PCR (IU/ml) & $101500(76750-164250)$ & $90000(75000-119000)$ & 0.339 \\
\hline
\end{tabular}

All data are presented as median (25th-75th).

$p$-value $<0.01$ is moderately significant, $p$-value $<0.001$ is highly significant.

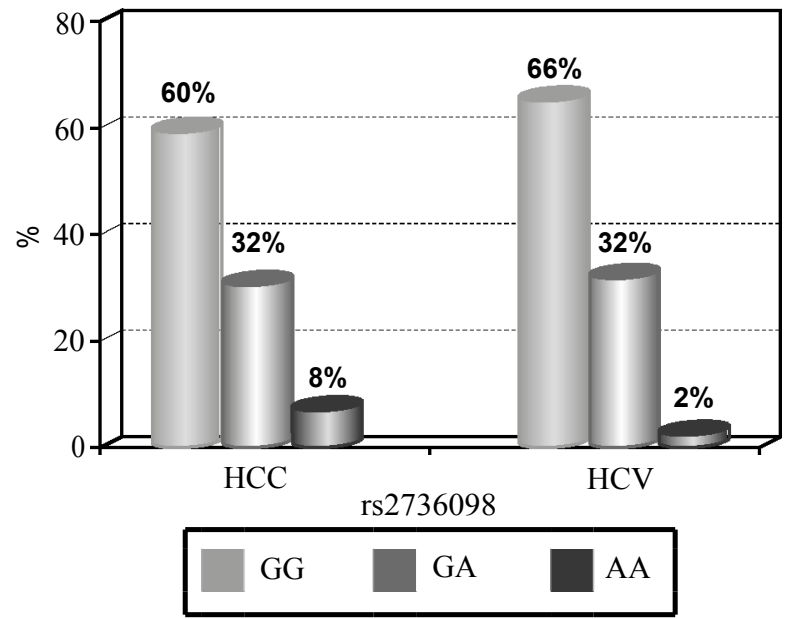

Fig. (1): Frequency distribution of rs2736098 genotypes in the two studied groups.

\section{Discussion}

Hepatocellular cancer is the sixth most common cancer and the third leading cause of death worldwide. It has a poor prognosis and the overall survival rates are $3-5 \%$. HCC has a mortality rate of $9.1 \%$ of global cancer [5]. The Telomerase Reverse Transcriptase (TERT) gene encodes the catalytic subunit of telomerase, which mediates pleiotropic effects, including the regulation of senescence and proliferation and plays an important role in carcinogenesis [3]. This study attempts to clarify the genetic predisposition to $\mathrm{HCC}$, focusing on the rs2736098 polymorphism. Because of its close association with telomere length, SNPs in TERT have been suggested as potential target genes for cancer therapy [10,11]. The rs2736098 (G>A) polymorphism, located in the second exon of TERT, is one of the most commonly investigated SNPs in the TERT gene, and its association with the risk of cancer was reported in various malignancies [13]. Although rs2736098 polymorphism leads to synonymous mutation or silent mutation, without amino acid changing (Asn305Asn), this SNP may influence telomerase activity and shorten telemere length because of the location within the gene regulatory elements and alteration of transcription factor binding $[15,18]$.

A homozygous GG genotype in SNP rs2736098 was high in both HCC and HCV compared to heterozygous GA genotype and variant AA genotype, and $\mathrm{G}$ allele compared to A allele was higher in $\mathrm{HCV}$ than $\mathrm{HCC}(82 \%$ versus $76 \%, p=)$. However, SNP rs2736098 showed no statistically significant difference between the two groups in the allele and genotype distribution.

To obtain a clear association between TERT rs2736098 and cancer risk, several meta-analyses have been performed. The first meta-analysis was conducted by Zhang et al., in 2013 [17], with 8 studies consisting of 8,070 cases and 10,239 controls. They claimed that no significant association was observed between TERT rs2736098 polymorphism and overall cancer risk.

However, after stratified by ethnicity, a significantly increased risk of cancers was shown among Asians. In another meta-analysis with 12 studies including 10044 cases and 12480 controls, Wu et al., [16] found that there was a borderline significant increased overall cancer risk conferred by rs2736098 [16]. In addition, such increased cancer risk was more obvious among lung cancer, bladder cancer, hospital-base design and Asians. Moreover, in another published meta-analysis included 19 studies with 12520 cases and 14968 controls. They 
found that GA/AA variant could contribute to increased risk of overall cancer. Their stratification analysis revealed that such association was more significant in Asians, lung cancer and HCC [9]

In a study conducted by Zhang et al., [17], four hundred patients with HCC and 400 non-cancer controls were genotyped to elucidate the potential association between TERT rs2736098 polymorphism and HCC risks. Compared with the controls, the patients with HCC had a lower frequency of $\mathrm{G} / \mathrm{G}$ genotype $(33.3 \%$ vs. $44.3 \%, p=0.001)$ and a higher frequency of G/A (51.5\% vs. $39.5 \%, p=$ $0.001)$. Allele genotypic frequencies in the patients differed from those of the controls $(p=0.040)$. The data of this study rs2736098 [A] allele contributed significantly to $\mathrm{HCC}$ risk in female patients $(\mathrm{OR}=$ $1.78,95 \% \mathrm{CI}, 1.17-2.72, p=0.007)$, patients with HCV infection $(\mathrm{OR}=2.89,95 \% \mathrm{CI}, 1.08-7.70, p=$ $0.031)$, non-drinker patients $(\mathrm{OR}=1.32,95 \% \mathrm{CI}$, $1.06-1.65, p=0.015)$, and patients not affected by HBV (OR=1.77, 95\% CI, 1.30-2.40, $p<0.001)$ [17]

In a study conducted by Zhou et al., [19], subgroup analysis based on cancer type indicated that the TERT rs2736098 (G>A) polymorphism was associated with HCC, the heterozygous and dominant genetic models supported an increased cancer susceptibility in the population carrying the rs2736098 A allele (GA vs. GG: OR $=1.595,95 \%$ $\mathrm{CI}=1.246-2.042, p=0.000$; and GA/AA vs. GG: $\mathrm{OR}=1.494,95 \% \mathrm{CI}=1.123-1.987, p=0.006)[19]$.

Yet, some limitations still exist and thus cautions are needed before interpreting the results obtained from the current study. First, ethnicity and small sample size. Second, the variation in HCC etiology might be discount as significant between-study heterogeneity was observed in some comparisons. Third the lack of other important information like environmental factors, age, drinking status, and gene-environment interactions.

\section{References}

1- ARMANIOS M. and BLACKBURN E.H.: The telomere syndromes. Nature Reviews Genetics, 13 (10): pp. 693704, 2012.

2- BERNAL A. and TUSELL L.: Telomeres: Implications for Cancer development. International Journal of Molecular Sciences, 19 (1): P. 294, 2018.

3- CLEAL K., NORRIS K. and BAIRD D.: Telomere Length Dynamics and the Evolution of Cancer Genome Architecture. International Journal of Molecular Sciences, 19 (2): p. 482, 2018.

4- De VITIS M., BERARDINELLI F. and SGURA A.: Telomere Length Maintenance in Cancer: At the Crossroad between Telomerase and Alternative Lengthening of
Telomeres (ALT). International Journal of Molecular Sciences, 19 (2): p. 606, 2018.

5- DENG H., SHI H., LEI J., HU Y., LI G. and WANG C.: A meta-analysis of contrast-enhanced ultrasound for small hepatocellular carcinoma diagnosis. Journal of Cancer Research and Therapeutics, 12 (8): p. 274, 2016.

6- GEWAID H., MESALAM A.A., IBRAHIM A.A., ABDEL SHAFY D.N., ABDEL SHAFY R.N., EMARA N., HAMDY S.M., GEWAID M. and BAHGAT M.M.: Establishment of a platform for molecular and immunological characterization of the RNA-dependent-RNA-polymerase NS5B of an Egyptian HCV isolate. Journal of Medical Virology, 2017.

7- JUNG S.W., PARK N.H., SHIN J.W., PARK B.R., KIM C.J., LEE J.E., SHIN E.S., KIM J.A. and CHUNG Y.H.: Prognostic impact of telomere maintenance gene poly morphisms on hepatocellular carcinoma patients with chronic hepatitis B. Hepatology, 59 (5): pp. 1912-20, 2014.

8- KANDA T., YOKOSUKA O. and OMATA M.: Hepatitis C Virus and Hepatocellular Carcinoma. Biology, 2: 30416, 2013.

9- LI T., XIAN Y., TIAN T., ZHUANG X. and CHU M.: New evidence of TERT rs 2736098 polymorphism and cancer risk: An updated meta-analysis. J. Buon., 21: 4917, 2016.

10- MELIN B.S., NORDFJÄLL K., ANDERSSON U. and ROOS G.: h TERT Cancer Risk Genotypes Are Associated With Telomere Length. Genetic Epidemiology, 36 (4): pp. 368-72, 2012.

11- NAN H., QURESHI A.A., PRESCOTT J., De VIVO I. and HAN J.: Genetic variants in telomere-maintaining genes and skin cancer risk. Human genetics. Mar. 1, 129 (3): 247-53, 2011.

12- OMAR A., ABOU-ALFA G.K., KHAIRY A. and OMAR H.: Risk factors for developing hepatocellular carcinoma in Egypt. Chin. Clin. Oncol., 2 (4): 43, 2013.

13- RAFNAR T., SULEM P., STACEY S.N., GELLER F., GUDMUNDSSON J., SIGURDSSON A. and BLÖNDAL T.: Sequence variants at the TERT-CLPTM1L locus associate with many cancer types. Nature Genetics, 41 (2): $221,2009$.

14- SHAY J.W. and WRIGHT W.E.: Role of telomeres and telomerase in cancer. In Seminars in cancer biology (Vol. 21, No. 6, pp. 349-53). Academic Press, 2011.

15- WANG J., LIU Q., YUAN S., XIE W., LIU Y., XIANG Y. and ZHANG Y.: Genetic predisposition to lung cancer: Comprehensive literature integration, meta-analysis, and multiple evidence assessment of candidate-gene association studies. Scientific Reports, 7 (1): 8371, 2017.

16- WU H., QIAO N., WANG Y., JIANG M., WANG S. WANG C. and HU L.: Association between the telomerase reverse transcriptase (TERT) rs2736098 polymorphism and cancer risk: Evidence from a case-control study of non-small-cell lung cancer and a meta-analysis. PLoS One, 8: e76372, 2013.

17- ZHANG C., TIAN Y.P., WANG Y., GUO F.H., QIN J.F. and NI H.: hTERT rs2736098 genetic variants and sus- 
ceptibility of hepatocellular carcinoma in the Chinese population: A case-control study. Hepatobiliary \& Pancreatic Diseases International, 12 (1): pp. 74-9, 2013.

18- ZHANG Y., ZHAO M., SHEN L., REN Y., SU L., LI X. and ZHOU B.: Genetic polymorphisms of TERT and CLPTM1L and risk of lung cancer: A case-control study in northeast Chinese male population. Medical oncology, 31 (7): 18, 2014.

19- ZHOU M., JIANG B., XIONG M. and ZHU X.: Association Between TERT rs2736098 Polymorphisms and Cancer Risk-A Meta-Analysis. Frontiers in Physiology, 9, 377, 2018.

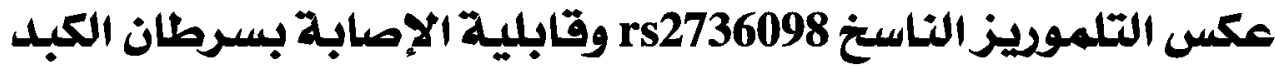 مع إلتهاب الكبد المزمن C فى المرضى المصريين}

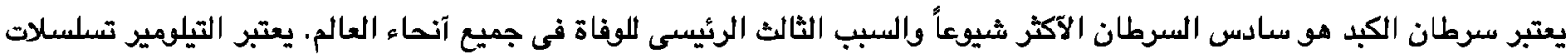

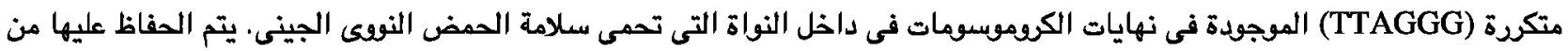

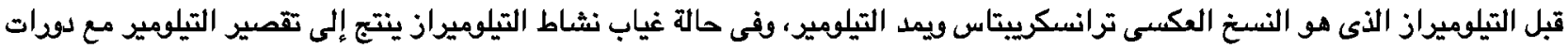
النسخ المتماثل.

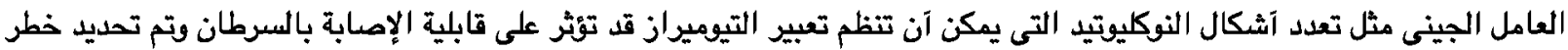

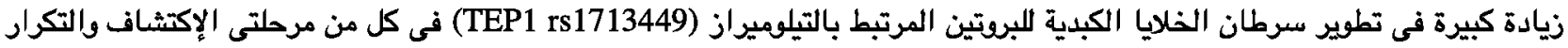

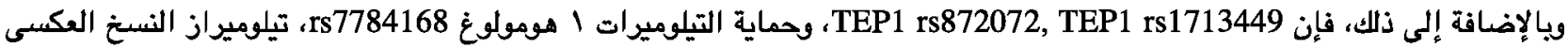

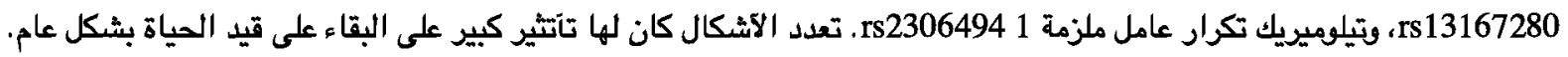

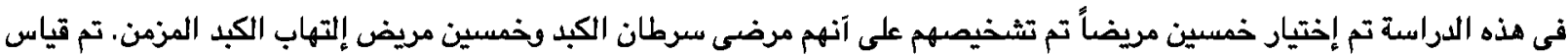

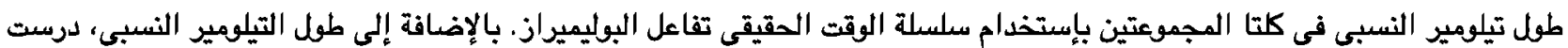

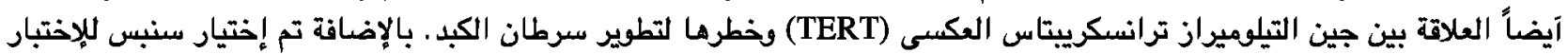
rs2736098. تم إجراء تحديد النمط الجينى بإستخدام تكمان سنب فحص التصني الآنماط الجينية.

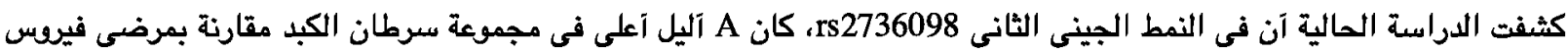

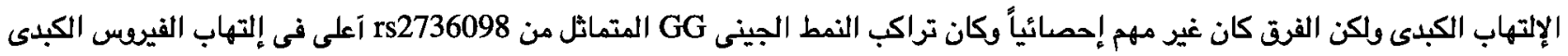

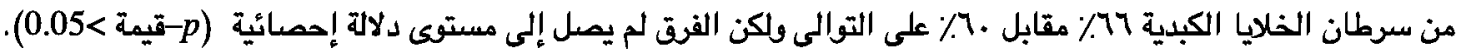

آجريت الدراسة الحالية للتحقق فيما إذا كانت التغيرات فى القيم المتوسطة على طول التيلومير النسبى ترتبط بكل من النمط الجينى ولكن مع عدم وجود إرتباط ذو دلالة إحصائية. 